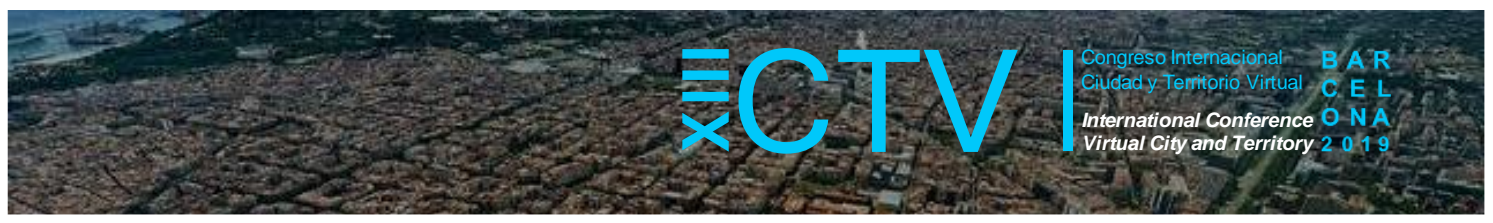

\title{
CONSECUENCIAS HIDROLÓGICAS DEL CAMBIO CLIMÁTICO EN ENTORNOS URBANOS
}

\author{
Casas-Castillo, M. Carmen ${ }^{1 *}$; Rodríguez-Solà, Raúl ${ }^{2}$; Lana, Xavier ${ }^{3}$; Serra, Carina ${ }^{4}$; Martínez, \\ M. Dolors ${ }^{5}$; Biere, Rolando ${ }^{6}$; Arellano, Blanca ${ }^{7}$ y Roca, Josep ${ }^{8}$
}

Remisión inicial: 2019-05-28; Remisión definitiva: 2019-09-22; Publicación: 2019-12-21

Citación: Casas-Castillo, M.C. et al. (2019). Consecuencias hidrológicas del cambio climático en entornos urbanos. En XIII CTV 2019 Proceedings: XIII International Conference on Virtual City and Territory: "Challenges and paradigms of the contemporary city": UPC, Barcelona, October 2-4, 2019. Barcelona: CPSV, 2019, p. 8291. E-ISSN 2604-6512. DOI http://dx.doi.org/10.5821/ctv.8291

\section{Resumen}

La posible intensificación del ciclo hidrológico debido al cambio climático y sus efectos en la hidrología deben tenerse en consideración con el fin de prevenir problemas futuros en los sistemas de drenaje urbano. Las curvas de intensidadduración-frecuencia (IDF), una importante herramienta utilizada en el diseño y construcción de diferentes estructuras hidrológicas para la gestión del agua, podrían verse alteradas por un supuesto aumento de las lluvias intensas debido al cambio climático (Rodríguez et al. 2014). Así como en otras latitudes se ha reportado un aumento de la precipitación total debido al calentamiento global (IPCC, 2007), en la zona mediterránea varios estudios apuntan una tendencia dominante decreciente en las últimas décadas (Pérez y Boscolo 2010). No ocurre así con la precipitación de carácter extremo o torrencial, para la cual se prevé un aumento, debido a una mayor ocurrencia de episodios lluviosos más cortos y de mayor intensidad (Christensen y Christensen 2003). La evaluación de estos cambios es especialmente interesante en las zonas urbanas con alta densidad de población, debido a la vulnerabilidad de sus complejos sistemas de alcantarillado frente a la lluvia torrencial (Casas et al. 2010, Rodríguez et al. 2013b).

Para describir matemáticamente las curvas IDF de un lugar, es posible utilizar una relación de escala relacionada con la propiedad fractal de invariancia de escala de la intensidad de lluvia (Menabde et al. 1999, Rodríguez-Solà et al. 2017). En el caso monofractal o de escala simple, esta relación de escala queda determinada por un único parámetro o exponente de escala $\beta$, cuyo valor parece estar relacionado con el régimen pluviométrico del lugar, o más en particular, con la irregularidad de la lluvia (Casas-Castillo et al. 2018b). Así, este parámetro toma valores cercanos a -1 para áreas en donde la lluvia suele ser muy irregular, con valores máximos aislados repentinos, mientras que toma valores más altos para áreas con un patrón de lluvia más regular. Es de esperar pues que la supuesta tendencia a la disminución en la precipitación anual y el aumento relativo en la precipitación extrema debido al cambio climático se vea reflejada en cambios en el comportamiento de escala de la lluvia, con valores del parámetro $\beta$ que tienden a disminuir. Con el fin de poner de manifiesto estos cambios, se han analizado series de precipitación diaria simuladas para el siglo XXI para dos ciudades densamente pobladas: Barcelona y Madrid. Estas series futuras fueron obtenidas a partir de varios modelos de circulación general y para diferentes escenarios de cambio climático: A1B, A2, B1 y B2 para Barcelona (114 series en total), y RCP2.6, RCP4.5, RCP6.0 y RCP8.5 para Madrid (213 series).

A pesar de la gran variabilidad de resultados obtenidos a partir de los modelos climáticos, es posible extraer algunas conclusiones con el suficiente grado de certidumbre. Para la precipitación total anual calculada a partir de las series futuras proyectadas se ha observado que, en Barcelona, dicha magnitud va a sufrir una disminución aproximada de un $5 \%$ en el último tercio del siglo XXI. En Madrid, en cambio, parece observarse un aumento poco significativo de la precipitación anual, siendo la incertidumbre en este caso muy alta.

En cuanto a las relaciones de escala simple obtenidas para la intensidad de lluvia futura en ambas ciudades, se ha obtenido una disminución significativa del exponente de escala $\beta$ a partir del segundo tercio del siglo XXI, lo cual es acorde con las previsiones de un posible aumento de las lluvias intensas en un futuro próximo debido al cambio

\footnotetext{
1 Departamento de Física, ESEIAAT, UPC, https://orcid.org/0000-0002-7507-6195; ${ }^{2}$ Departamento de Física, ETSEIB, UPC, https://orcid.org/0000-0002-9623-894X; ${ }^{3}$ Departamento de Física, ETSEIB, UPC, https://orcid.org/0000-00023298-9234; ${ }^{4}$ Departamento de Física, ETSEIB, UPC, https://orcid.org/0000-0003-3803-2414; ${ }^{5}$ Departamento de Física, ETSEIB, UPC, https://orcid.org/0000-0001-8200-183X; ${ }^{6}$ Departamento de Tecnología de la Arquitectura, CPSV, ETSAB, UPC, https://orcid.org/0000-0003-1872-7104; ${ }^{7}$ Departamento de Tecnología de la Arquitectura, CPSV, ETSAB, UPC, https://orcid.org/0000-0001-7128-3667 y ${ }^{8}$ Departamento de Tecnología de la Arquitectura, CPSV, ETSAB, UPC, https://orcid.org/0000-0003-3970-6505 * Correo de contacto: m.carmen.casas@upc.edu
} 


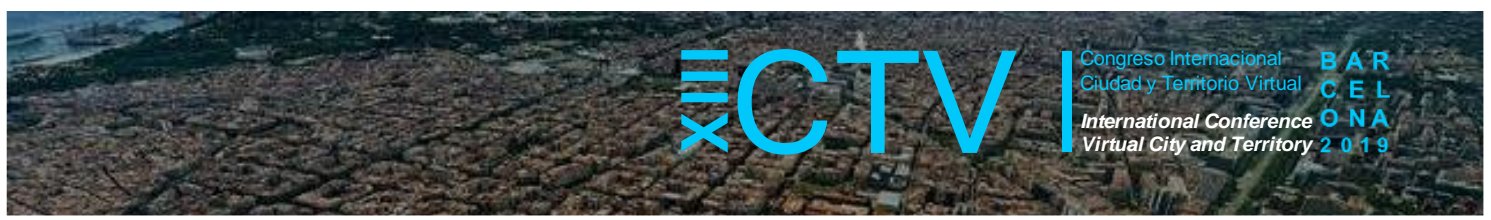

climático. Estos resultados indican un probable incremento en la frecuencia de las lluvias de carácter torrencial y el consecuente aumento del riesgo de inundación en ambas ciudades, por lo que debería tenerse en consideración en el diseño de su infraestructura hidráulica, siendo recomendable una mejora de sus redes de alcantarillado y de sus sistemas de drenaje acorde a estas previsiones.

\section{Abstract}

The possible intensification of the hydrological cycle due to climate change and its effects on hydrology should be taken into account in order to prevent future problems in urban drainage systems. The intensity-duration-frequency (IDF) curves, an important tool used in the design and construction of different hydrological structures for water management, could be altered by a supposed increase in heavy rainfall due to climate change (Willems et al. 2011, Rodríguez et al. 2014). Whereas in other latitudes an increase in total precipitation due to global warming has been reported (IPCC, 2007), in the Mediterranean area several studies point to a decreasing dominant trend in recent decades (Pérez and Boscolo 2010). This is not the case with extreme or torrential precipitation, for which an increase is expected, due to a greater occurrence of shorter and more intense rainy episodes (Christensen and Christensen 2003). The evaluation of these changes is especially interesting in urban areas with high population density, due to the vulnerability of their complex sewage systems to torrential rain (Casas et al. 2010, Rodríguez et al. 2013b).

To describe mathematically the IDF curves of a place, it is possible to use a scaling relationship related to the fractal property of scale invariance of rainfall intensity (Menabde et al. 1999, Rodríguez-Solà et al. 2017). In the monofractal case or simple scale, this scaling relationship is determined by a single parameter or scaling exponent $\beta$, whose value seems to be related to the rainfall pattern of the place, or more particularly, to the irregularity of the rain (Casas-Castillo et al. 2018b). Thus, this parameter takes values close to -1 for areas where rain is usually very irregular, with sudden maximum isolated values, while it takes higher values for areas with a more regular rainfall pattern. It is to be expected that the supposed tendency of the annual precipitation to decrease and the relative increase in extreme precipitation due to climate change will be reflected in changes in the rainfall scaling behavior, with values of parameter $\beta$ that tend to decrease. In order to highlight these changes, simulated daily precipitation series for the 21 st century have been analyzed for two densely populated cities: Barcelona and Madrid. These future series were obtained from several general circulation models and for different climate change scenarios: A1B, A2, B1 and B2 for Barcelona (114 series in total), and RCP2.6, RCP4.5, RCP6.0 and RCP8.5 for Madrid (213 series).

In spite of the great variability of results obtained from the climatic models, it is possible to draw some conclusions with the sufficient degree of certainty. For the total annual precipitation calculated from the projected future series, it has been observed that, in Barcelona, this magnitude will suffer an approximate decrease of $5 \%$ in the last third of the 21 st century. In Madrid, on the other hand, there seems to be a little significant increase in annual rainfall, being the uncertainty very high in this case.

Regarding the simple scaling relationships obtained for the intensity of future rainfall in both cities, a significant decrease in the scaling exponent $\beta$ has been obtained from the second third of the 21 st century, which is consistent with the forecasts of a possible increase of heavy rains in the near future due to climate change. These results indicate a probable increase in the frequency of torrential rains and the consequent increase in the risk of flooding in both cities, so it should be taken into consideration in the design of its hydraulic infrastructure, being recommended an according improvement of its networks of sewage system and its drainage systems.

Palabras Clave: cambio climático; curvas IDF; lluvia extrema; comportamiento de escala

Key words: climate change; IDF curves; extreme rainfall; scaling behavior

\section{Introducción}

El aumento global de la temperatura del planeta conllevará sin duda alteraciones permanentes en el ciclo hidrológico, por lo que cabe esperar cambios en la distribución espacial de la precipitación, con aumentos en algunas áreas y disminuciones en otras. Aun cuando no llegase a cambiar la cantidad total de precipitación, la frecuencia de días lluviosos o la intensidad de la precipitación podrían experimentar fuertes variaciones en respuesta a un aumento de la concentración atmosférica de vapor de agua. Incluso un pequeño desplazamiento en el valor 


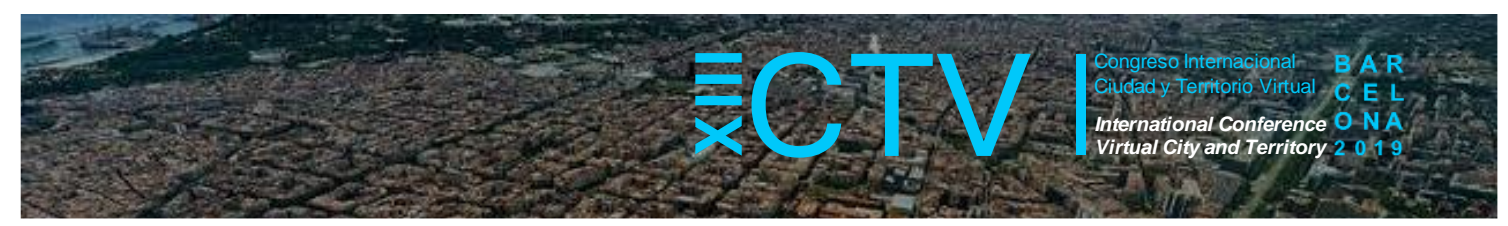

medio de la precipitación podría acarrear cambios importantes en la distribución de valores extremos y, por tanto, posibles variaciones en la frecuencia de episodios extremos de precipitación.

Contrariamente a lo que ocurre en las regiones de latitud alta o media del mundo, en las que se ha reportado un incremento en la precipitación total, varios estudios muestran una tendencia decreciente dominante durante las últimas décadas en el área mediterránea (Intergovernmental Panel on Climate Change, IPCC, 2007). De este modo, el informe de evaluación regional de cambio climático elaborado por investigadores de la red CLIVAR-España (Pérez y Boscolo, 2010) llegó a la conclusión de que la precipitación anual en la Península lbérica en las últimas tres décadas ha disminuido significativamente en comparación con los años 1960 y 1970, siendo al final del invierno la disminución más importante observada. Sin embargo, la fuerte variabilidad interanual y la falta de series que se remonten a principios del siglo XX impiden afirmar que la precipitación haya descendido de forma generalizada a mínimos históricos. En conjunto, la señal antropogénica en la precipitación no ha emergido de forma evidente por encima de la variabilidad natural.

A pesar de esta disminución en la precipitación total, se ha observado, en cambio, un aumento de las precipitaciones extremas. Mientras que las simulaciones para el siglo XXI que utilizan modelos climáticos también pronostican descensos importantes en la precipitación total en el área mediterránea, algunas proyecciones de modelos regionales muestran un posible aumento de las lluvias torrenciales (Christensen y Christensen, 2003), con episodios de precipitación más cortos y más intensos. Para la región del Mediterráneo noroccidental, Hertig y Jacobeit (2008) encontraron un aumento de la precipitación en invierno para el período 2071-2100, bajo el escenario B2 (uno de los escenarios de emisiones de gases de efecto invernadero del SRES, Special Report on Emission Scenarios, publicado por el IPCC http://www.ipcc.ch/ipccreports/sres/emission/, que modelizan las emisiones futuras en base a diferentes patrones de desarrollo social, económico, político y tecnológico), mientras que en otoño y primavera encontraron una disminución. El posible aumento en las condiciones anticiclónicas en las estaciones de transición (otoño y primavera) en la región del Mediterráneo podría ser la causa de esta reducción en la precipitación. El aumento de la precipitación en invierno podría estar causado por un aumento en las condiciones ciclónicas, en particular en diciembre y enero.

En el estudio de Buonomo et al. (2007) se encontró que los valores extremos de precipitación aumentaran tanto al aumentar el periodo de retorno de la precipitación como al disminuir la duración considerada: para la región de Europa en promedio, la lluvia diaria con un periodo de retorno de 20 años se incrementará en un 18\% a finales del siglo XXI, mientras que para un periodo de retorno de 2 años el incremento medio será sólo del $13 \%$. Y estos aumentos son inferiores cuando se consideran duraciones de más de 24 horas.

En general, lo que se observa es un aumento de las precipitaciones extremas en oposición a una reducción en la precipitación media anual. Esto está indicando un cambio en el clima futuro, con una reducción sustancial de los episodios de precipitación ligera o moderada y, en cambio, un aumento de los episodios de lluvia intensa. En términos del factor de cambio climático (cociente entre el valor estimado de precipitación futura y el actual), el estudio de Larsen et al. (2009) estimó valores generalmente por encima de 1 para las precipitaciones horarias en Europa en el período de 2071 a 2100 bajo el escenario de cambio climático A2. 


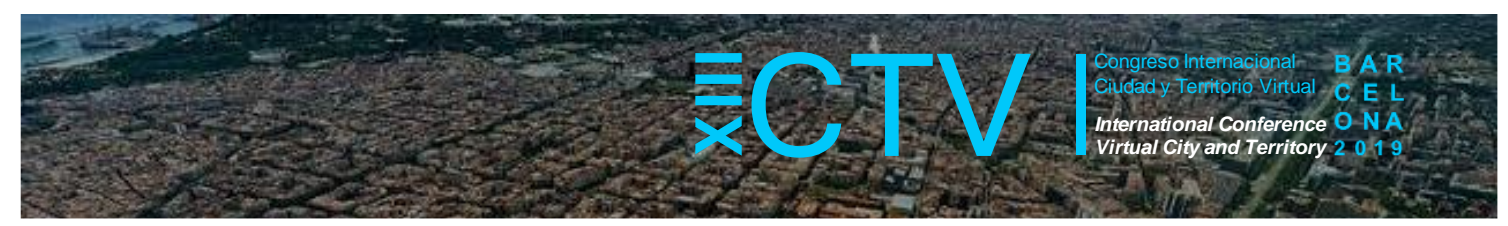

Para la zona del mediterráneo occidental, el factor de cambio climático calculado resultó cercano a 1,2 para períodos de retorno entre 5 y 100 años.

La evaluación de la posible influencia del cambio climático en la intensidad de las lluvias extremas es muy interesante en el área mediterránea, especialmente en zonas urbanas densamente pobladas con complejos sistemas de alcantarillado, generalmente vulnerables a las Iluvias torrenciales. En el estudio de Rodríguez et al. (2014) se estimaron los cambios futuros a esperar en las actuales curvas de intensidad-duración-frecuencia (IDF) de Barcelona, una importante herramienta útil en el diseño y construcción de diferentes estructuras hidrológicas para la gestión del agua. En dicho estudio se obtuvo que, para el último tercio del siglo XXI, bajo los escenarios climáticos A1B, A2 y B2, se espera un aumento de al menos un $4 \%$ en la precipitación diaria con un período de retorno superior a 20 años, mientras que para la precipitación horaria esperada el aumento resultó, para casi todos los escenarios y períodos considerados, todavía un poco más alto que la precipitación diaria correspondiente. Las mayores diferencias entre la futura lluvia horaria y diaria estimada se encontraron en el segundo tercio del siglo bajo los escenarios A1B (la precipitación horaria un $8 \%$ superior, en media, a la precipitación diaria) y A2 (un $9 \%$ superior). Este mayor aumento esperado de las precipitaciones para duraciones más cortas (horarias) frente a las diarias, es acorde con un posible aumento de la lluvia de carácter intenso o torrencial con respecto al resto.

Para el estudio de Rodríguez et al. (2014), la precipitación diaria futura en Barcelona se estimó a partir de series simuladas por la Fundación para la investigación del Clima (FIC), haciendo uso de una técnica de reducción de escala estadística (downscaling espacial) basada en el marco conceptual presentado por Ribalaygua et al. (2013). La estimación de la precipitación horaria se realizó en Rodríguez et al. (2014) utilizando un método de downscaling temporal basado en la propiedad fractal de invariancia de escala de la lluvia. En el trabajo que aquí se presenta, se ha extendido este análisis de escala a las series diarias de lluvia simuladas por la FIC para la ciudad de Madrid, presentándose también la variación esperada en la precipitación anual de ambas ciudades.

\section{Metodología}

\subsection{Downscaling espacial}

Los modelos de circulación general (CGMs) proporcionan proyecciones climáticas futuras a gran escala. Para inferir información a escala regional a partir de estas proyecciones se suelen utilizar diferentes técnicas de reducción de escala (downscaling espacial), entre ellas las que hacen servir una aproximación estadística (Wilby et al., 2004, Imbert y Benestad, 2005), para las cuales los predictando de alta resolución, como la lluvia, se obtienen aplicando relaciones climáticas previamente identificadas con los predictores a gran escala, como por ejemplo los flujos geostróficos atmosféricos.

Las series de precipitación diaria futura en Barcelona analizadas por Rodríguez et al. (2014) fueron simuladas por la FIC a partir de la información a gran escala proporcionada por cinco modelos de circulación general: cuatro provenientes del Fourth Assessment Report AR4 (EGMAM, CNCM3, ECHAM5 y BCM2) del IPCC (2007) y un quinto proveniente del Third Assessment Report AR3 (CGCM2). Se consideraron tres escenarios de emisiones de gases de efecto invernadero, el A1B, A2 y B1, y el período de control 1951-1999, para los modelos 


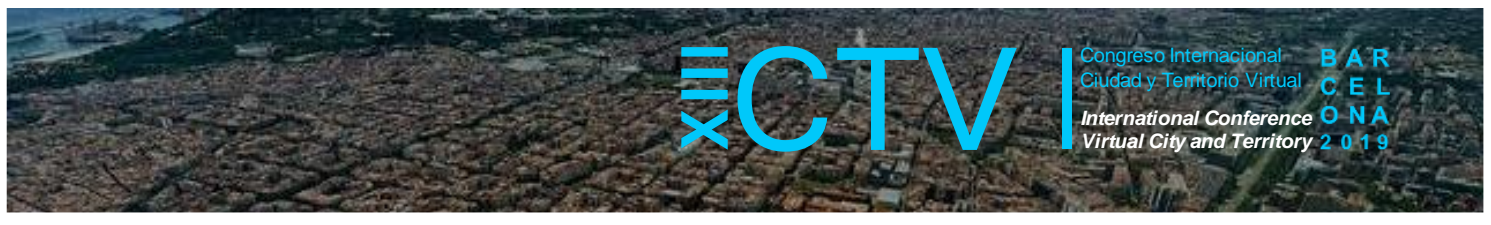

EGMAM, CNCM3, ECHAM5 y BCM2, y dos escenarios, A2 y B2, y el período de control 19611999, para el modelo CGCM2 (IPCC, 2000). Esta información climática fue reducida espacialmente de escala para generar las series simuladas correspondientes a seis estaciones pluviométricas que la Agencia Estatal de Meteorología (AEMET) tiene instaladas en el área metropolitana de Barcelona (véase figura 1), de las cuales se disponía, además, de más de 20 años de datos diarios de precipitación observada. Para la ciudad de Madrid la FIC simuló series de precipitación diaria para tres estaciones AEMET: Retiro, Torrejón de Ardoz y Getafe (véase figura 2), utilizando nueve modelos de circulación general, cuatro escenarios de clima futuro (RCP2.6, RCP4.5, RCP6.0 y RCP8.5) y el periodo histórico de control 1951-2005.

Figura 1. Situación de las estaciones del área de Barcelona

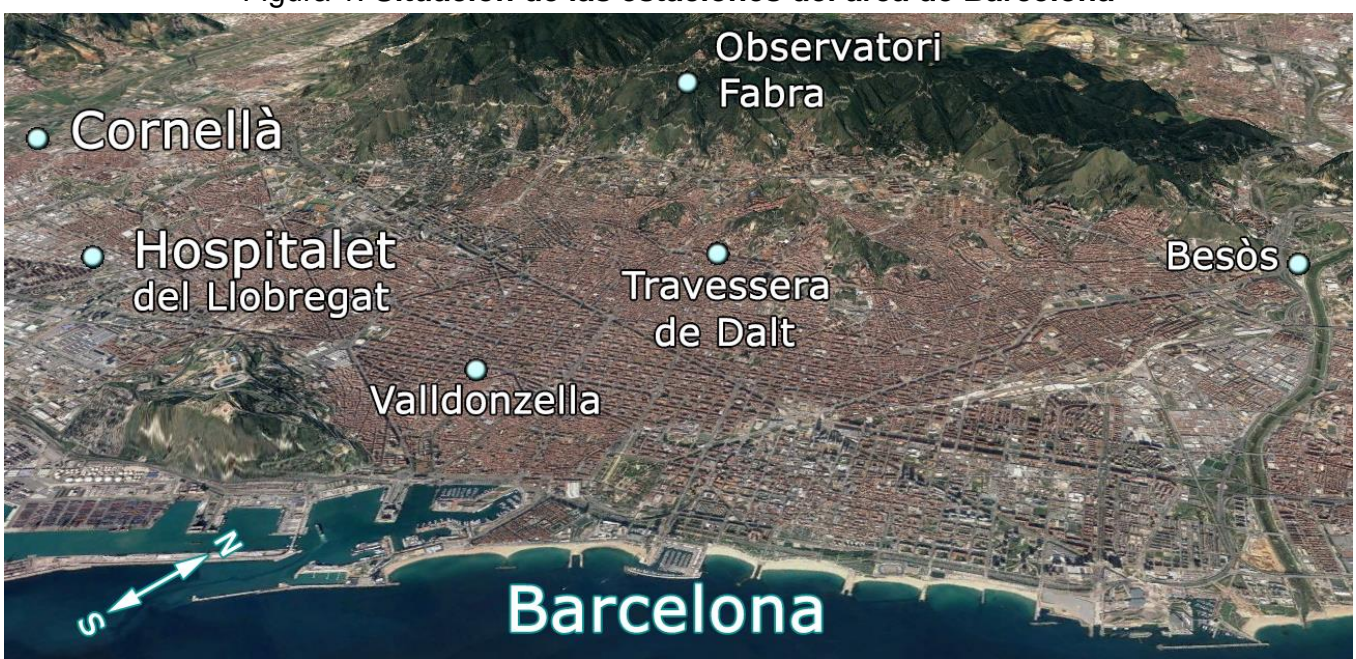

Fuente: GoogleEarth.

Figura 2. Situación de las estaciones del área de Madrid

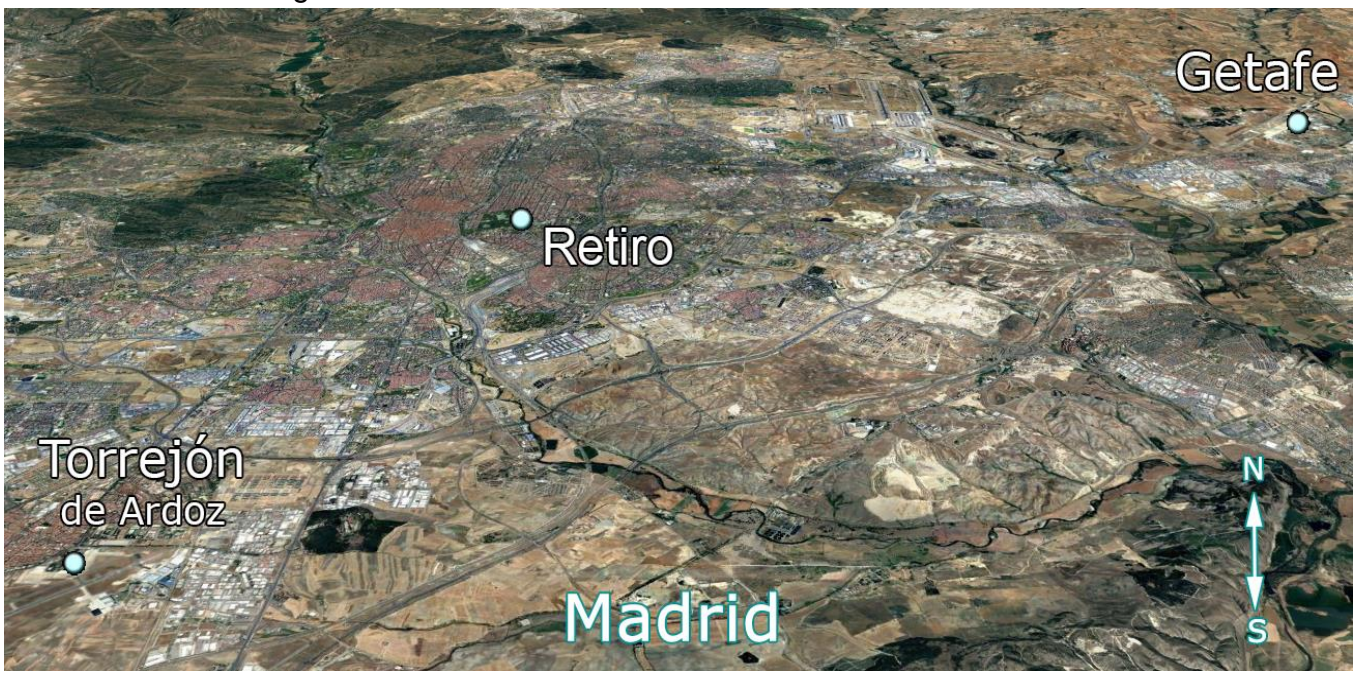

Fuente: GoogleEarth.

El método de reducción de escala utilizado (Ribalaygua et al., 2013) estima los campos meteorológicos de alta resolución en superficie para un día en concreto en dos pasos: el primer paso es una técnica de análogos (Zorita y von Storch, 1999), y en la segunda etapa la información de alta resolución en superficie (por ejemplo, la precipitación) se estima mediante 


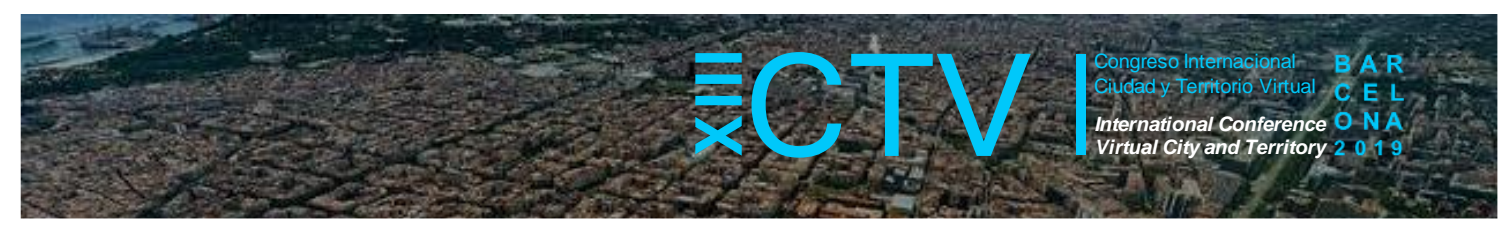

una aproximación probabilística. Este procedimiento supone que las relaciones entre los predictores (variables atmosféricas a gran escala) y los predictando (variables de superficie de pequeña escala) no variarán en el futuro bajo condiciones de cambio climático. En el primer paso, igual que en otros métodos de reducción de escala por análogos, para cada día de salida de los modelos climáticos se buscan las situaciones meteorológicas más similares observadas en las series históricas pasadas, utilizando los flujos geostróficos atmosféricos como el dato relevante como criterio de similitud. Estos flujos determinan el forzamiento sinóptico que provoca que el aire ascienda o descienda, dando lugar a la formación de nubes y la precipitación. Además, el flujo en $1000 \mathrm{hPa}$ proporciona información sobre la dirección del viento en superficie, dato muy útil para estudiar el efecto de la topografía en la nubosidad y la distribución espacial de precipitación. El segundo paso en el procedimiento de Ribalaygua et al. (2013) es la determinación de la relación entre las variables atmosféricas a gran escala y las variables de superficie a menor escala, y su validación con las observaciones. La precipitación futura se acaba estimando como la precipitación media observada para los días que han resultado más similares al día concreto que se quiere simular.

\subsection{Downscaling temporal}

Las curvas de intensidad-duración-frecuencia (curvas IDF) son una herramienta de considerable interés para ingenieros e hidrólogos. Las relaciones matemáticas que se usan con más frecuencia para describir estas curvas suelen ser empíricas, a veces en forma de una ecuación generalizada para la intensidad de lluvia I $(\mathrm{t}, \mathrm{T})$, válida para todas las duraciones $\mathrm{t}$ y los períodos de retorno $T$ considerados. Esta ecuación generalmente tiene la forma generalizada $a(T) / b(t)$, donde $a(T)$ y $b(t)$ son funciones independientes entre sí. Aunque la función $a(T)$ se puede encontrar empíricamente (Casas et al. 2004), existen otros enfoques que buscan poner de manifiesto ciertas características de las series como, por ejemplo, Koutsoyiannis et al. (1998), que propone el uso de una función de probabilidad estadística de la intensidad máxima de lluvia para determinar dicha función. Otro enfoque posible consiste en tener en consideración la propiedad fractal de invariancia de escala de las series de lluvia, más concretamente, obteniendo relaciones de escala de los momentos estadísticos de las series de máximos anuales (Burlando y Rosso 1996; Menabde et al. 1999). En esta línea existen diversos trabajos que han dado lugar a la obtención de una relación analítica para las curvas IDF teniendo en cuenta el comportamiento de escala (Yu et al. 2004; Desramaut 2008; Bara et al. 2010; Aronica y Freni 2005; Pérez-Zanón et al. 2016; Rodríguez-Solà et al. 2017; CasasCastillo et al. 2018a y 2018b).

Muchos procesos atmosféricos, entre ellos la generación de lluvia, actúan en un amplio rango temporal dando lugar a fenómenos con auto-similitud, es decir, que se ven iguales independientemente de la escala temporal en la que se observan. Este tipo de procesos puede considerarse de tipo fractal, con propiedades que se pueden expresar con leyes potenciales del parámetro de escala $\lambda$, que es la razón $t / t_{0}$ entre dos duraciones $t$ y $t_{0}$ dentro de un mismo régimen de escala. En general, la auto-similitud fractal de los procesos naturales tiene una naturaleza estadística; por lo tanto, las propiedades de escala de la lluvia pueden expresarse mediante relaciones estadísticas (Schertzer y Lovejoy 1987; Gupta y Waymire 1990; Schertzer y Lovejoy 2011). Las series de máximos anuales de intensidad de lluvia suelen satisfacer relaciones de escala, en el sentido de que la distribución de probabilidad de la intensidad 


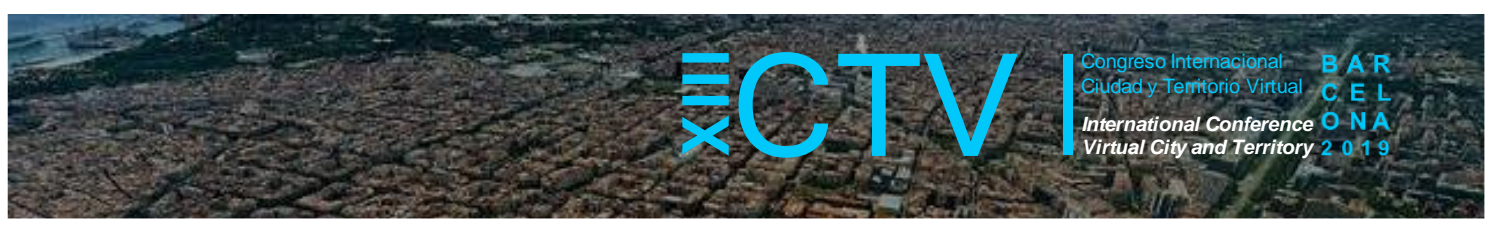

máxima anual para una duración $t, I_{t}$, y la distribución para otra duración $t_{0}=\lambda t, I_{\lambda t}$, pueden relacionarse mediante un factor que es una función potencial del parámetro de escala $\lambda$. Esta relación suele expresarse como la ecuación (1),

$$
\mathrm{I}_{\mathrm{t}}^{\text {dist }}=\lambda^{\beta} \mathrm{I}_{\lambda \mathrm{t}}
$$

en la cual $\beta$ es un parámetro de escala. El símbolo $\stackrel{\text { dist }}{=}$ de la ecuación (1) indica la igualdad probabilística entre las dos distribuciones a ambos miembros de la ecuación (Gupta and Waymire 1990; Yu et al. 2004), y por tanto también satisfarán está igualdad los momentos estadísticos de ambas distribuciones, así como sus cuantiles y el resto de características estadísticas. En términos de los momentos estadísticos de orden q de la intensidad de lluvia para una duración $t,\left\langle l_{t}^{a}\right\rangle$ (véase ecuación (2)), la relación de escala viene dada por la ecuación (3):

$$
\begin{aligned}
\left\langle l_{t}^{q}\right\rangle & =\frac{\sum_{i=1}^{n} l_{t_{i}}^{q}}{n} \\
\left\langle l_{t}^{q}\right\rangle & =\lambda^{\beta a}\left\langle l_{\lambda t}^{q}\right\rangle
\end{aligned}
$$

El exponente $\beta q$ es el valor que corresponde a una función de escala general $\mathrm{K}(\mathrm{q})$ cuando ésta resulta ser lineal (caso monofractal). Cuando esta función de escala es no lineal lo que se tiene es un multifractal (Rodríguez et al. 2013a). El procedimiento más sencillo para determinar el parámetro de escala $\beta$ a partir de datos diarios consiste en calcular los momentos estadísticos de series de máximos anuales obtenidas por agregación de las series diarias, con cantidades de lluvia de 2, 3, 4... días, para diferentes valores del orden q, y ajustar por regresión lineal el logaritmo de dichos momentos frente al logaritmo de la duración t para cada valor de $q$. Las líneas rectas obtenidas, cada una de ellas con una pendiente de valor $\beta q$, indican invariancia de escala.

La igualdad para los cuantiles estadísticos de las distribuciones a ambos miembros de la ecuación (1) implica que es posible encontrar una relación de escala para otros valores extremos de intensidad de lluvia, por ejemplo la intensidad para una duración $t$ con un período de retorno $\mathrm{T}$, es decir, las curvas IDF I $(\mathrm{t}, \mathrm{T})$. Esta relación de escala puede ser la ecuación (4) si se utiliza 24 horas como la duración de referencia $t_{0}$ :

$$
\mathrm{I}(\mathrm{t}, \mathrm{T})=\left(\frac{\mathrm{t}}{24}\right)^{\beta} \mathrm{I}(24, \mathrm{~T})
$$

Una vez conocido el parámetro de escala $\beta$, la ecuación (4) puede utilizarse para obtener valores IDF subdiarios a partir de los valores diarios $I(24, T)$, con la suposición de que la relación de escala simple también se satisface para las duraciones subdiarias, lo cual sólo puede considerarse como una aproximación. Rodríguez-Solà et al. (2017) aplicaron esta técnica para reproducir de manera satisfactoria las, bien conocidas, curvas IDF de tres localidades de la PI: Barcelona, Madrid y el Observatorio del Ebro.

Por su definición, los valores del parámetro de escala simple $\beta$ han de ser superiores a -1 . Este valor límite $\beta=-1$ correspondería a una muestra de lluvia con máximos anuales de lluvia 


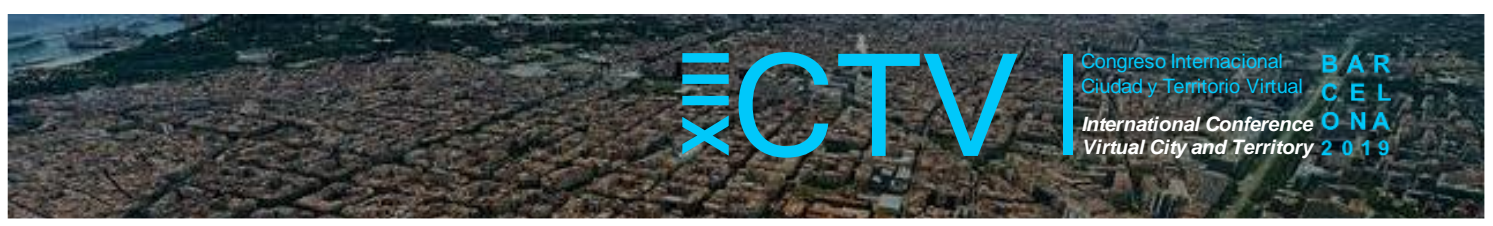

aislados. Esto es, en el caso de lluvia diaria, tener un valor máximo para un día específico $\mathrm{P}_{1}$ rodeado por días secos, sin precipitación. Por tanto, el proceso de agregación da lugar a series para las cuales la precipitación para $n$ días, $P_{n}$, es la misma. En términos de la intensidad de precipitación esto sería $I_{n}=I_{1} / n$, que corresponde al caso $\beta=-1$ si lo comparamos con la relación de escala para $q=1$ (la media), que es $I_{1} / n=n^{\beta} I_{1}$. El hipotético caso opuesto sería el de una muestra totalmente regular para la cual todos los días llueve lo mismo (y para todas las agregaciones se tiene la misma cantidad de precipitación), lo que implica la misma intensidad para todas las duraciones, y por tanto $\beta=0$. Para la lluvia real este parámetro suele oscilar entre el valor -1 y un valor próximo a $\beta=-0.5$.

Parece razonable esperar cierta relación entre los valores del parámetro $\beta$ y la regularidad de las series de lluvia, con los valores más bajos próximos a -1 para áreas en donde la llueve suele ser muy irregular, con valores máximos súbitos aislados, y valores más altos para áreas lluviosas con un patrón lluvioso más regular. Rodríguez-Solà et al. (2017), por ejemplo, encontraron una concordancia general entre la distribución espacial del parámetro $\beta$ para la PI y la precipitación anual media, con valores altos entre -0.55 y -0.66 en las zonas más lluviosas (Galicia, Cantabria...) y bajos entre -0.84 y -0.92 para las zonas más áridas (Levante sur), con algunas discrepancias relacionadas con el tipo de precipitación contribuyendo a las cantidades más altas de precipitación y la proporción de lluvia convectiva en el total. En concordancia con este resultado, Casas-Castillo et al. (2018b) encontraron también una buena correlación entre el parámetro $\beta$ y la lluvia media anual en Cataluña, con los valores más bajos al norte (Pirineos, sobre todo occidentales, y la zona más al noroeste, Viella con cierta influencia atlántica), y los más altos en las zonas más secas del sudoeste (Lérida). En este estudio no se encontró correlación entre $\beta$ y la longitud geográfica, pero sí con la latitud ( $\beta$ aumentando hacia el norte), con la altitud ( $\beta$ aumentando con la elevación) y, debido a la influencia del mar Mediterráneo, con la distancia a la línea de costa ( $\beta$ aumentando al aumentar la distancia). En el estudio de Casas-Castillo et al. (2018b) también se analizó la evolución temporal del parámetro $\beta$ durante el siglo $X X$ y principios del $X X I$, encontrándose una ligera tendencia decreciente, especialmente significativa en los Pirineos occidentales.

También se obtuvo una tendencia decreciente significativa de $\beta$ durante la segunda mitad del siglo XX en la serie centenaria registrada en el Observatorio del Ebro (1905-2016). Ambos resultados son compatibles con el esperado aumento de las lluvias extremas en relación al descenso de la precipitación anual.

En el trabajo que aquí se presenta se va a exponer la evolución temporal esperada de la precipitación anual durante el siglo XXI, calculada a partir de las series futuras de lluvia simuladas tanto para la localidad de Barcelona como la de Madrid, así como la evolución temporal futura del parámetro $\beta$ en ambas ciudades.

\section{Resultados y conclusiones}

En concordancia con los estudios que pronostican una posible disminución futura de la precipitación anual en la zona mediterránea debido al cambio climático, el análisis de las series 


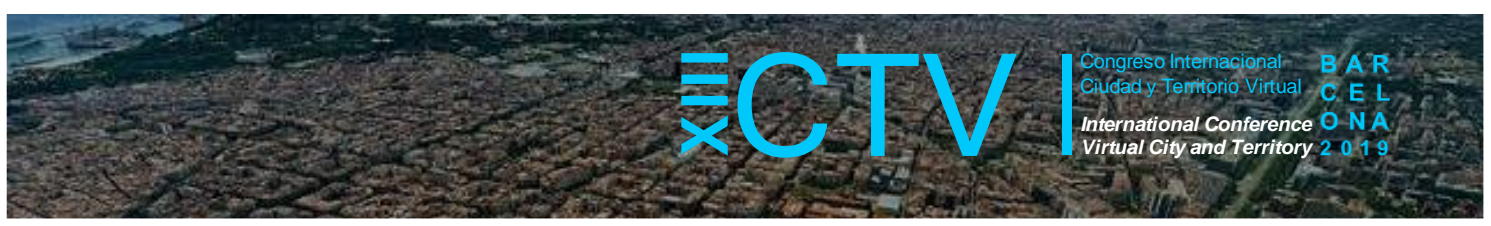

de lluvia futura simuladas para Barcelona (Rodríguez et al. 2014) dio como promedio una disminución continua de la precipitación anual en dicha ciudad, bajo todos los escenarios de cambio climático considerado, previéndose una disminución aproximada de al menos un $5 \%$ para el último tercio del siglo XXI para tres de los escenarios climáticos analizados (A1B, A2 i B1).

La figura 3 muestra la evolución de la precipitación anual en Barcelona para estos tres escenarios climáticos obtenida como la media de todos los resultados obtenidos para cada modelo climático y cada estación meteorológica. Se muestra como la variación relativa de la precipitación media calculada respecto de la correspondiente al período de referencia (1971 2000 en este caso). Las sombras coloreadas indican el error de dispersión de los resultados, para todos los modelos y todos los observatorios analizados, donde el límite superior es el percentil 90 y el inferior el percentil 10. Este resultado es comparable con los obtenidos por Sillman et al. (2013) y por Kovats et al. (2014) para el sur de Europa utilizando los escenarios RCP asociados al quinto informe IPCC.

Figura 3. Evolución temporal prevista de la precipitación anual en Barcelona

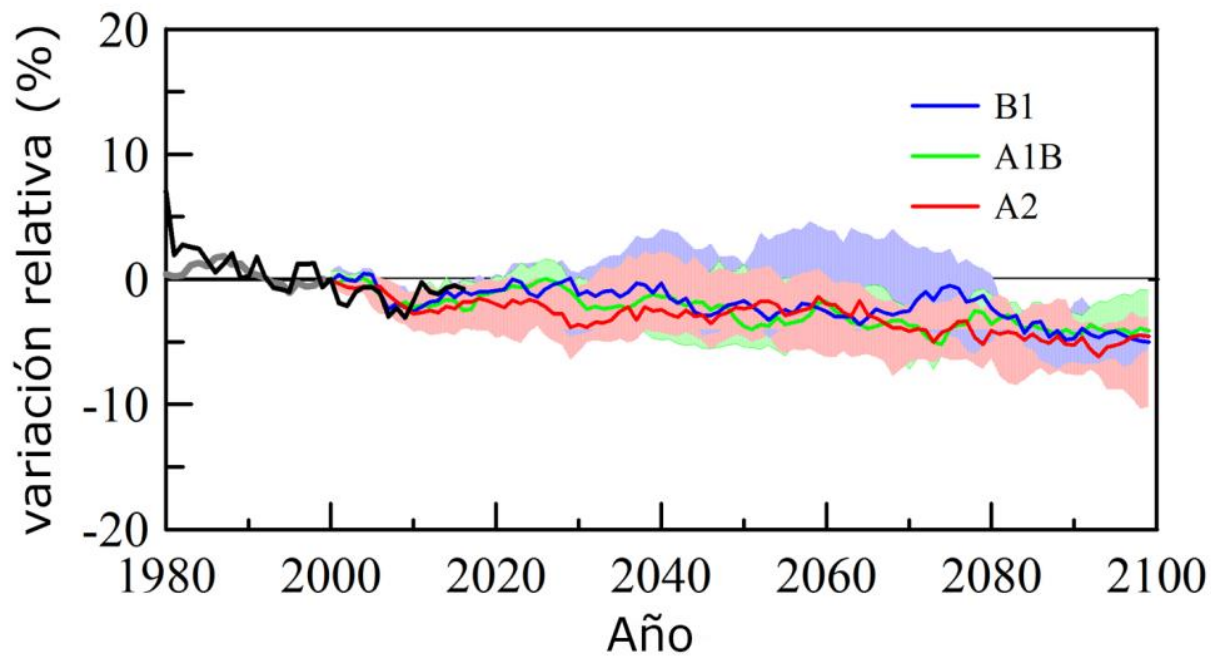

Fuente: Elaboración propia.

En cambio, para la ciudad de Madrid, la previsión respecto a la precipitación anual futura ha dado un resultado algo más incierto. Bajo tres de los cuatro escenarios climáticos analizados, RCP2.6, RCP4.5 y RCP8.5, la precipitación anual parece mantenerse sin cambios para la mayor parte del siglo XXI, y experimentar un ligero aumento inferior al $5 \%$ en el último tercio de siglo, con un error de dispersión que no acaba de definirse en cuanto al aumento o la disminución (véase figura 4).

Bajo el escenario RCP6.0 las previsiones dan una ligera disminución de la precipitación anual hacia el final de siglo, aunque la estadística bajo este escenario es menos significativa y no es comparable a la estadística bajo los otros tres escenarios dado que sólo se obtuvieron previsiones para 5 de los 9 modelos de circulación general empleados. 


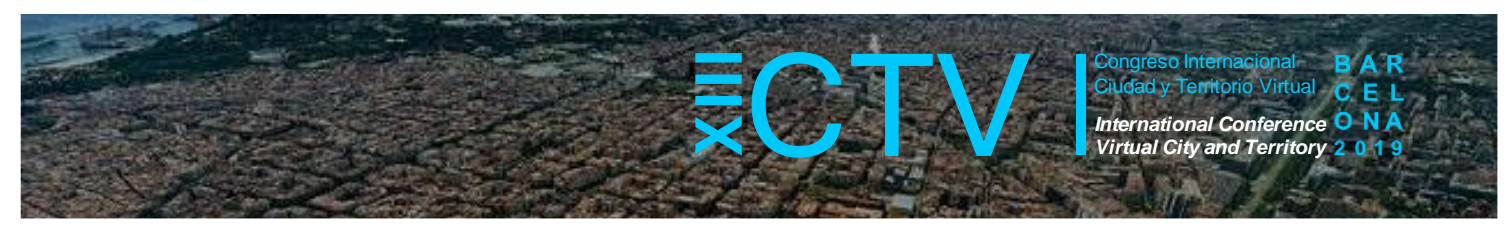

Figura 4. Evolución temporal prevista de la precipitación anual en Madrid

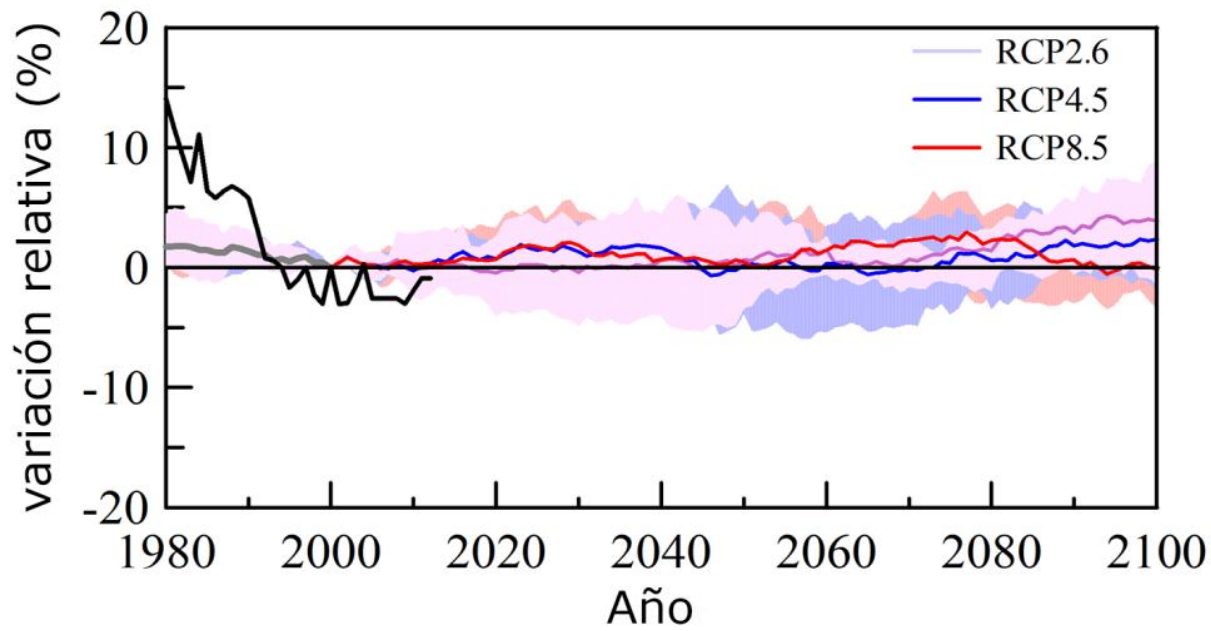

Fuente: Elaboración propia.

Así, si la dispersión de resultados para Barcelona permite estimar que el valor medio de precipitación anual podría disminuir desde el actual de $612 \mathrm{~mm}$ a un valor aproximado de 580 $\mathrm{mm}$ a finales del siglo XXI, para Madrid no es posible hacer una estimación fiable a partir de estas simulaciones. Atendiendo a los resultados para dos de los escenarios más significativos estadísticamente, RCP 2.6 y RCP8.5, podría haber un ligero aumento de la precipitación anual en Madrid hacia finales de siglo, que actualmente es de $450 \mathrm{~mm}$, para situarse entre los $460 \mathrm{y}$ los $470 \mathrm{~mm}$.

En cuanto a la evolución temporal futura de los valores del parámetro de escala $\beta$, estos se calculan tomando intervalos de 30 años sucesivos que varían el rango temporal en 1 año. Las previsiones, tanto para Madrid como para Barcelona, indican una disminución de este parámetro a lo largo del siglo XXI, acorde con una evolución hacia un patrón de lluvia más árido, con picos de intensidad de lluvia más aislados. En el caso de Madrid, la combinación de resultados del posible ligero aumento de la precipitación anual conjuntamente con la disminución del parámetro de escala indica un posible aumento de las lluvias de carácter extremo.

El parámetro de escala actual de Madrid, de valor -0.71, calculado con datos de la estación del Retiro (Rodríguez-Solà et al. 2017) ya de por sí indica un patrón de lluvias ligeramente más regular que el correspondiente al Observatorio Fabra de Barcelona, de valor -0.78 . Las previsiones en cuanto al futuro indican que dicho parámetro podría disminuir hacia valores cercanos a -0.75 en Madrid (véase figura 5), es decir, el patrón lluvioso seguiría siendo algo más regular que el actual de Barcelona. Las previsiones futuras para Barcelona (figura 6) indican que el parámetro de escala posiblemente disminuirá por debajo de -0.80 , acercándose incluso a -0.85 bajo alguno de los escenarios, valor que en la PI solamente se observa en la actualidad al sur de Levante en la PI y en lbiza en las Islas Baleares (Rodríguez-Solà et al. 2017). 
Figura 5. Evolución temporal del parámetro de escala $\beta$ para Madrid

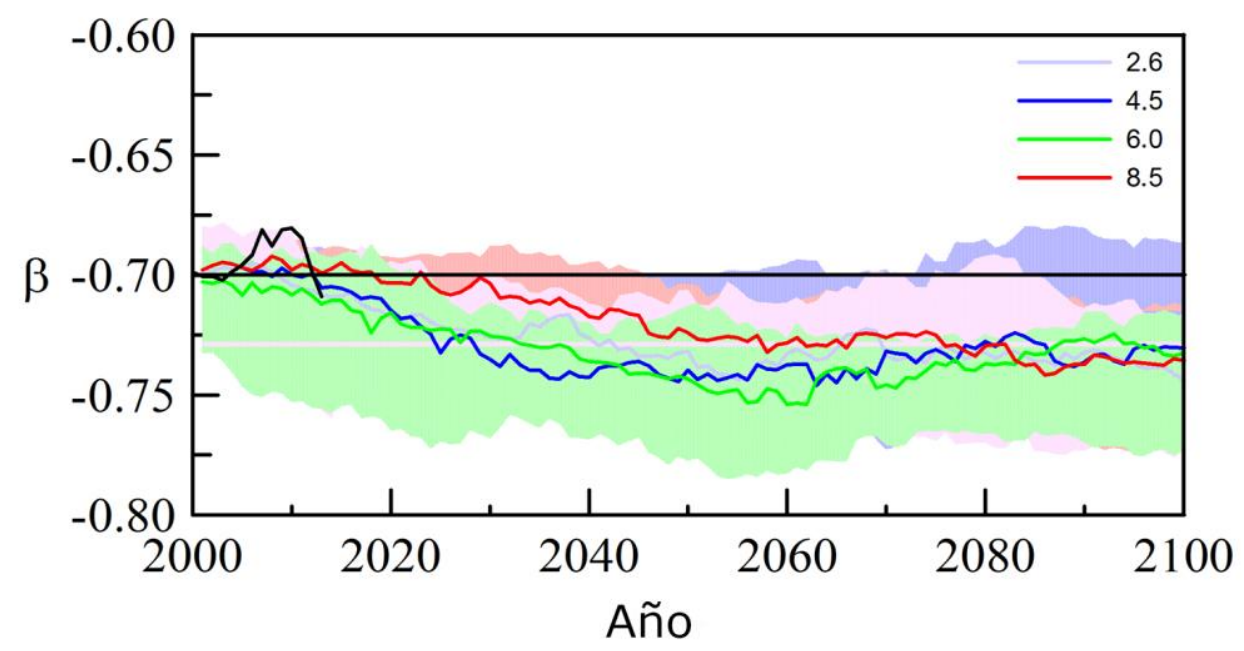

Fuente: Elaboración propia.

Figura 6. Evolución temporal del parámetro de escala $\beta$ para Barcelona

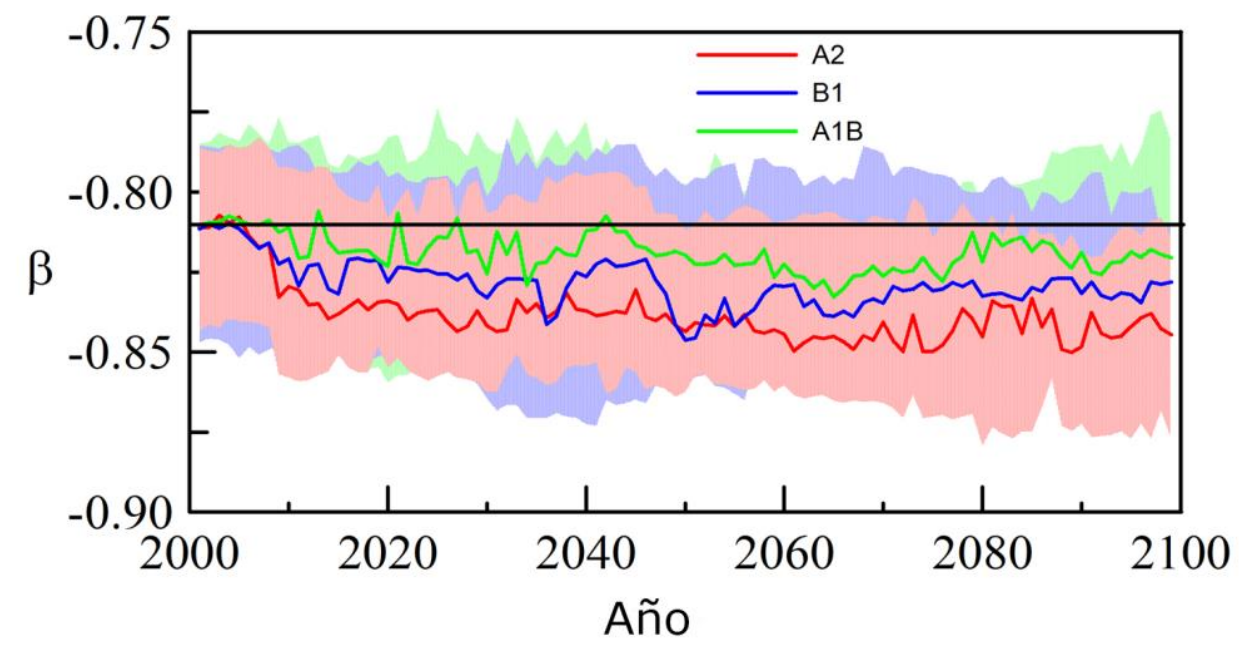

Fuente: Elaboración propia.

A partir de las series futuras simuladas para Barcelona, Rodríguez et al. (2014) encontraron que, bajo los escenarios climáticos A1B y A2, las precipitaciones diarias futuras correspondientes a períodos de retorno superiores a 10 años, es decir las más extremas, podrían aumentar entre un 3\% y un $7 \%$ para el último tercio de siglo. Por tanto, si según las curvas IDF actuales de Barcelona (Casas et al. 2004) después de observar una lluvia que recoja al menos $107 \mathrm{~mm}$ en 24 horas hay que esperar una media de 10 años para observar otra de similares características (calculado a partir del(24 h, 10 a)), atendiendo a las estimaciones futuras, tendríamos con esa misma frecuencia lluvias algo más abundantes, y por tanto habría que esperar una media de entre 7 y 8.5 años para observar esa misma cantidad de precipitación en 24 horas. Cuando se tiene en consideración el parámetro de escala $\beta$ para estimar las precipitaciones horarias, mientras que en la actualidad la correspondiente al 


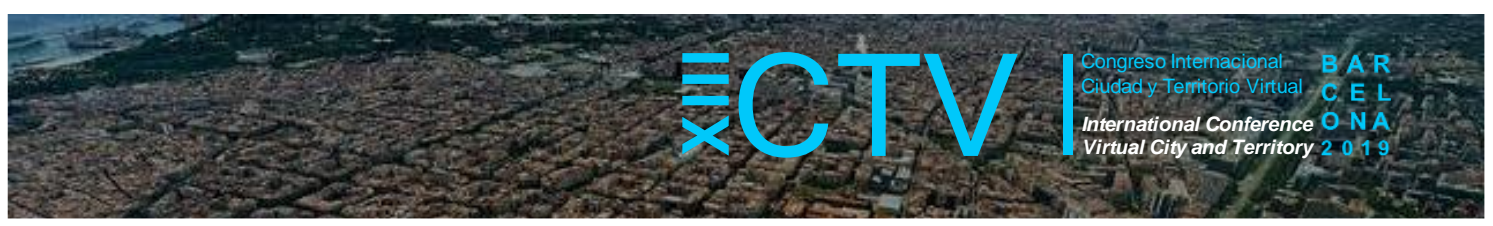

período de retorno de 10 años es de $60 \mathrm{~mm}$, bajo todos los escenarios de cambio climático analizados para el último tercio de siglo, es de esperar para esa misma frecuencia lluvias horarias entre un $4 \%$ y un $13 \%$ superiores, y por tanto para la misma cantidad de precipitación de $60 \mathrm{~mm}$ en 1 hora, el período de retorno disminuye hasta valores entre 5 y 8 años. Es decir, Rodríguez et al. (2014) encontraron mayores aumentos en la lluvia horaria futura de Barcelona que en la diaria.

Aplicando este mismo análisis para Madrid también se han encontrado, en general, mayores aumentos en la lluvia horaria futura que en la diaria. Promediando los resultados dada la gran variabilidad observada, se obtienen, por ejemplo, los siguientes resultados para un período de retorno $T$ de 20 años:

- Se estima un aumento de la lluvia diaria de lo que queda de siglo XXI en el $61 \%$ de los casos estudiados, siendo superior al $5 \%$ de aumento en el $51 \%$ de los casos. Comparando con Barcelona el aumento estimado en la lluvia diaria también resulta en el $61 \%$ de los casos, pero sólo en un $38 \%$ este aumento supera el $5 \%$.

- Se estima un aumento de la lluvia horaria en el $78 \%$ de los casos, siendo este aumento superior al $10 \%$ en un $63 \%$ de los casos. Mientras que en Barcelona la lluvia horaria aumenta en un $68 \%$ de los casos y el aumento supera el $10 \%$ para un $45 \%$.

- $\quad$ En promedio se espera un aumento de entre el 2 y el $8 \%$ de la lluvia diaria en Madrid bajo la mayoría de escenarios. Para este período de retorno, en Barcelona este aumento no supera el $5 \%$.

- Se estima un aumento promedio de la lluvia horaria en Madrid superior al 9\%, siendo de casi un $20 \%$ para el tercer tercio de siglo, bajo los escenarios climáticos RCP2.6, RCP4.5 y RCP8.5. Para este período de retorno, en Barcelona este aumento se encuentra entre el $5 \%$ y el $14 \%$ según el escenario climático considerado.

Así pues, a pesar de la gran variabilidad de resultados obtenidos a partir de los modelos climáticos, es posible extraer algunas conclusiones con el suficiente grado de certidumbre. Para la precipitación total anual calculada a partir de las series futuras proyectadas se ha observado que, en Barcelona, dicha magnitud va a sufrir una disminución aproximada de un $5 \%$ en el último tercio del siglo XXI. En Madrid, en cambio, parece observarse un aumento poco significativo de la precipitación anual, siendo la incertidumbre en este caso muy alta.

Atendiendo al análisis de la evolución temporal del parámetro que determina las relaciones de escala simple obtenidas para la intensidad de lluvia futura en ambas ciudades, se ha obtenido una disminución significativa del exponente de escala $\beta$ a partir del segundo tercio del siglo XXI y por lo tanto es de esperar mayores aumentos en la lluvia horaria futura que en la diaria. Este mayor aumento esperado de las precipitaciones para duraciones cortas es acorde con las previsiones de un posible aumento de las lluvias intensas o incluso de carácter torrencial en un futuro próximo debido al cambio climático y el consecuente aumento del riesgo de inundación en ambas ciudades, por lo que debería tenerse en consideración en el diseño de su infraestructura hidráulica, siendo recomendable una mejora de sus redes de alcantarillado y de sus sistemas de drenaje acorde a estas previsiones. 


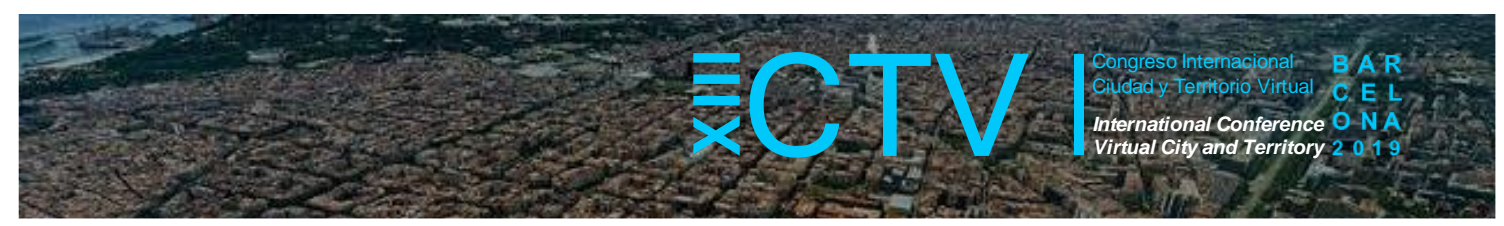

Agradecimientos: Este estudio ha sido financiado en parte por el Canal de Isabel II Gestión para el Plan Maestro de Drenaje de la Comunidad de Madrid, y en parte por CETaqua y $R+i$ Alliance para el proyecto SW0801-phase2. Los autores agradecen a Clavegueram de Barcelona (CLABSA), a Barcelona Cicle de l'aigua (BCASA) y a la Fundación para la Investigación del Clima (FIC) por haber proporcionado parte de los datos de lluvia utilizados.

Contribuciones de los autores: El desarrollo general del trabajo ha sido llevado a cabo de manera conjunta por los dos primeros autores. La primera autora se ha encargado de la confección del artículo y las gráficas, en la confección de las cuales ha contribuido también el segundo autor, responsable de realizar además la mayor parte de los cálculos del trabajo. El resto de autores han contribuido en la revisión y mejora del artículo.

Conflicto de Intereses: Los autores declaran que no hay conflicto de intereses.

\section{Bibliografía}

Aronica, G. T. y Freni, G. (2005). Estimation of sub-hourly DDF curves using scaling properties of hourly and sub-hourly data at partially gauged site. Atmospheric Research, 77(1-4): 114-123. DOI: 10.1016/i.atmosres.2004.10.025

Bara, M.; Gaál, L.; Kohnová, S.; Szolgay, J. y Hlavčová, K. (2010). On the use of the simple scaling of heavy rainfall in a regional estimation of IDF curves in Slovakia. Journal of Hydrology and Hydromechanics, 58(1): 49-63. DOI: 10.2478/v10098-010-0006-0

Buonomo, E.; Jones, E.; Huntingford, C. y Hammaford, J. (2007). On the robustness of changes in extreme precipitation over Europe from two high resolution climate change simulations. The Quarterly Journal of the Royal Meteorological Society, 133(622): 65-81. DOI: 10.1002/aj.13

Burlando, P. y Rosso, R. (1996). Scaling and multiscaling models of depth-duration-frequency curves for storm precipitation. Journal of Hydrology, 187(1-2): 45-64. DOI: 10.1016/S0022$\underline{1694(96) 03086-7}$

Casas, M. C.; Codina, B.; Redaño, Á. y Lorente, J. (2004). A methodology to classify extreme rainfall events in the western Mediterranean area. Theor. Appl. Climatol., 77(3-4): 139-150. DOI: $10.1007 / \mathrm{s} 00704-003-0003-\mathrm{x}$

Casas, M. C.; Rodríguez, R. y Redaño, Á. (2010). Analysis of extreme rainfall in Barcelona using a microscale rain gauge network. Meteorol. Appl., 17:117-123. DOI: $\underline{10.1002 / \text { met.166 }}$

Casas-Castillo, M. C.; Rodríguez-Solà, R.; Navarro, X.; Russo, B.; Lastra, A.; González, P. y Redaño, Á. (2018a). On the consideration of scaling properties of extreme rainfall in Madrid (Spain) for developing a generalized intensity-duration-frequency equation and assessing probable maximum precipitation estimates. Theor. Appl. Climatol., 131(1-2): 573-580. DOI: $\underline{10.1007 / \mathrm{s} 00704-016-1998-0}$

Casas-Castillo, M. C.; Llabrés-Brustenga, A.; Rius, A.; Rodríguez-Solà, R. y Navarro, X. (2018b). A single scaling parameter as a first approximation to describe the rainfall pattern of a place: application on Catalonia. Acta Geophys., 66(3): 415-424. DOI: 10.1007/s11600-018$\underline{0122-5}$ 


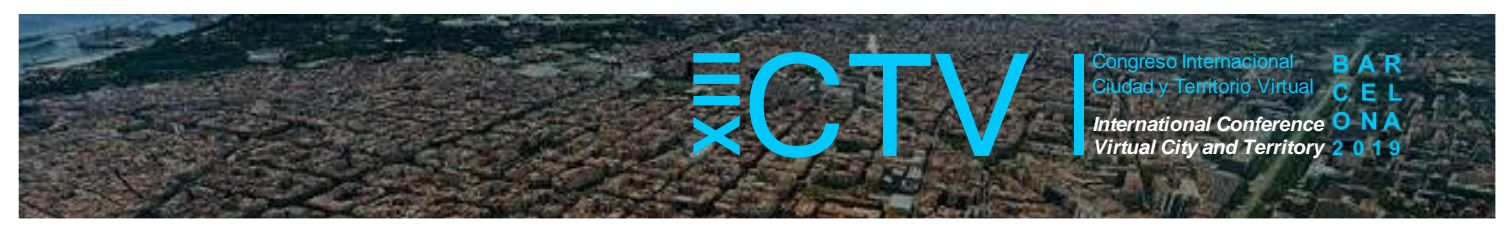

Christensen, J. H. y Christensen, O. B. (2003). Severe summertime flooding in Europe. Nature, 421: 805-806. DOI: 10.1038/421805a

Desramaut, N. (2008). Estimation of intensity Duration Frequency Curves for Current and Future Climates, Thesis of Master. Department of Civil Engineering and Applied Mechanics. McGill University, Montreal, Quebec, Canada. Recuperado de digitool.library.mcgill.ca/thesisfile40816.pdf

Gupta, V. K. y Waymire, E. (1990). Multiscaling properties of spatial rainfall and river flow distributions. Journal of Geophysical Research, Atmospheres, 95(D3): 1999-2009. DOI: 10.1029/JD095iD03p01999

Hertig, E. y Jacobeit, J. (2008). Assessments of Mediterranean precipitation changes for the 21st century using statistical downscaling techniques. Int. J. Climatol., 28: 1025-1045. DOI: 10.1002/joc. 1597

Imbert, A. y Benestad, R. E. (2005). An improvement of analog model strategy for more reliable local climate change scenarios. Theor. Appl. Climatol., 82(3-4):245-255. DOI: 10.1007/s00704$\underline{005-0133-4}$

IPCC (2007). Fourth Assessment Report of the Intergovernmental Panel on Climate Change. University Press: Cambridge, UK.

Kovats, R. S.; Valentini, R.; Bouwer, L. M.; Georgopoulou, E.; Jacob, D.; Martin, E.; Rounsevell, M. y Soussana, J. F. (2014). Europe. En V. R. Barros, C. B Field, D. J. Dokken, M. D. Mastrandrea, K. J. Mach, T. E. Bilir, M. Chatterjee, K. L. Ebi, Y. O. Estrada, R. C. Genova, B. Girma, E. S. Kissel, A. N. Levy, S. MacCracken, P. R. Mastrandrea y L. L. White (eds.). Climate Change 2014: Impacts, Adaptation, and Vulnerability. Part B: Regional Aspects. Contribution of Working Group II to the Fifth Assessment Report of the Intergovernmental Panel on Climate Change (pp. 1267-1326). Cambridge, Reino Unido, y New York, EEUU: Cambridge University Press.

Pérez, F. F. y Boscolo, R. (2010). Clima en España: Pasado, presente y futuro. Informe de Evaluación del Cambio climático Regional. Red Temática CLIVAR-España. Recuperado de http://hdl.handle.net/10261/23600

Pérez-Zanón, N.; Casas-Castillo, M. C.; Rodríguez-Solà, R.; Peña. J. C.; Rius, A.; Solé, J. G. y Redaño. Á. (2016). Analysis of extreme rainfall in the Ebre Observatory (Spain). Theor. Appl. Climatol., 124(3-4): 935-944. DOI: 10.1007/s00704-015-1476-0

Larsen, A. N.; Gregersen, I. B.; Christensen, O. B.; Linde, J. J. y Mikkelsen, P. S. (2009). Potential future increase in extreme one-hour precipitation events over Europe due to climate change. Water Science and Technology, 60: 2205-2216. DOI: 10.2166/wst.2009.650

Menabde, M.; Seed, A. y Pegram, G. (1999). A simple scaling model for extreme rainfall. Water Resour. Res., 35(1):335-339. DOI: 10.1029/1998WR900012

Ribalaygua, J.; Torres, L.; Pórtoles, J.; Monjo, R.; Gaitán, E. y Pino, M. R. (2013). Description and validation of a two-step analogue/regression downscaling method. Theor. Appl. Climatol., 114(1-2):253-369. DOI: $\underline{10.1007 / \mathrm{s} 00704-013-0836-\mathrm{x}}$ 


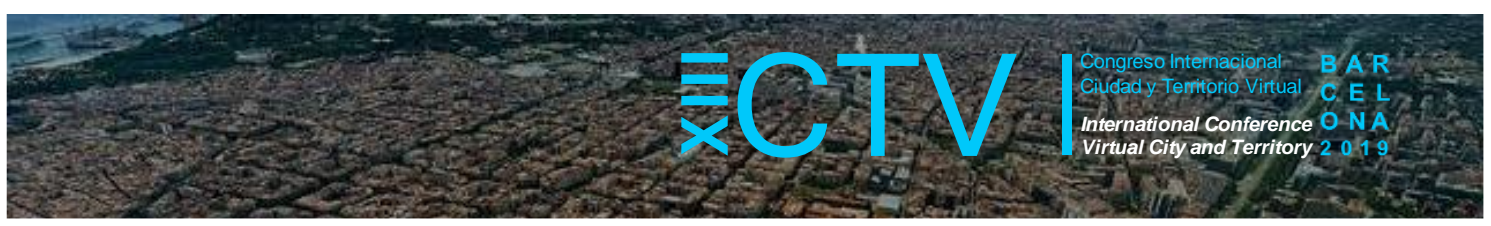

Rodríguez, R.; Casas, M. C. y Redaño, Á. (2013a). Multifractal analysis of the rainfall time distribution on the metropolitan area of Barcelona (Spain). Meteorol. Atmos. Phys., 121(3-4): 181-187. DOI: $10.1007 / \mathrm{s} 00703-013-0256-6$

Rodríguez, R.; Navarro, X.; Casas, M. C. y Redaño, Á. (2013b). Rainfall spatial organization and areal reduction factors in the metropolitan area of Barcelona (Spain). Theor. Appl. Climatol., 114(1):1-8. DOI: $10.1007 / \mathrm{s} 00704-012-0818-4$

Rodríguez, R.; Navarro, X.; Casas, M. C.; Ribalaygua, J.; Russo, B.; Pouget, L. y Redaño, Á. (2014). Influence of climate change on IDF curves for the metropolitan area of Barcelona (Spain). Int. J. Climatol., 34: 643-654. DOI: 10.1002/joc.3712

Rodríguez-Solà, R.; Casas-Castillo, M. C.; Navarro, X. y Redaño, Á. (2017). A study of the scaling properties of rainfall in Spain and its appropriateness to generate intensity-durationfrequency curves from daily records. Int. J. Climatol., 37(2):770-780. DOI: $10.1002 /$ joc.4738

Schertzer, D. y Lovejoy, S. (1987). Physical modeling and analysis of rain and clouds by anisotropic scaling multiplicative processes. Journal of Geophysical Research, Atmospheres, 92(D8): 9693-9714. DOI: 10.1029/JD092iD08p09693

Schertzer, D. y Lovejoy, S. (2011). Multifractals, generalized scale invariance and complexity in geophysics. International Journal of Bifurcation and Chaos, 21(12): 3417-3456. DOI: $\underline{10.1142 / S 0218127411030647}$

Sillmann, J.; Kharin, V. V.; Zhang, X.; Zwiers, F. W. y Bronaugh, D. (2013) Climate extremes indices in the CMIP5 multimodel ensemble: Part 1. Model evaluation in the present climate. Journal of Geophysical Research, Atmospheres, 118(4): 1716-1733. DOI: 10.1002/igrd.50203

Wilby, R. L.; Charles, S. P.; Zorita, E.; Timbal, B.; Whetton, P. y Mearns, L. O. (2004). The guidelines for use of climate scenarios developed from statistical downscaling methods. Supporting material of the Intergovernmental Panel on Climate Change (IPCC), prepared on behalf of Task Group on Data and Scenario Support for Impacts and Climate Analysis (TGICA). Recuperado de http://www.narccap.ucar.edu/doc/tgica-guidance-2004.pdf

Yu, P. S.; Yang, T. C. y Lin, C. S. (2004). Regional rainfall intensity formulas based on scaling property of rainfall. Journal of Hydrology, 295(1-4): 108-123. DOI: 10.1016/i.jhydrol.2004.03.003

Zorita, E. y von Storch, H. (1999). The analog method as a simple statistical downscaling technique: comparison with more complicated methods. Journal of Climate, 12: 2474-2489. DOI: 10.1175/1520-0442(1999)012\%3C2474:TAMAAS\%3E2.0.CO;2 